Document downloaded from:

http://hdl.handle.net/10251/180377

This paper must be cited as:

Xu, SZ.; Chen, JL.; Benítez López, J. (2021). Partial orders based on the CS decomposition. Ukrainian Mathematical Journal. 72(8):1294-1313. https://doi.org/10.1007/s11253-02001851-5

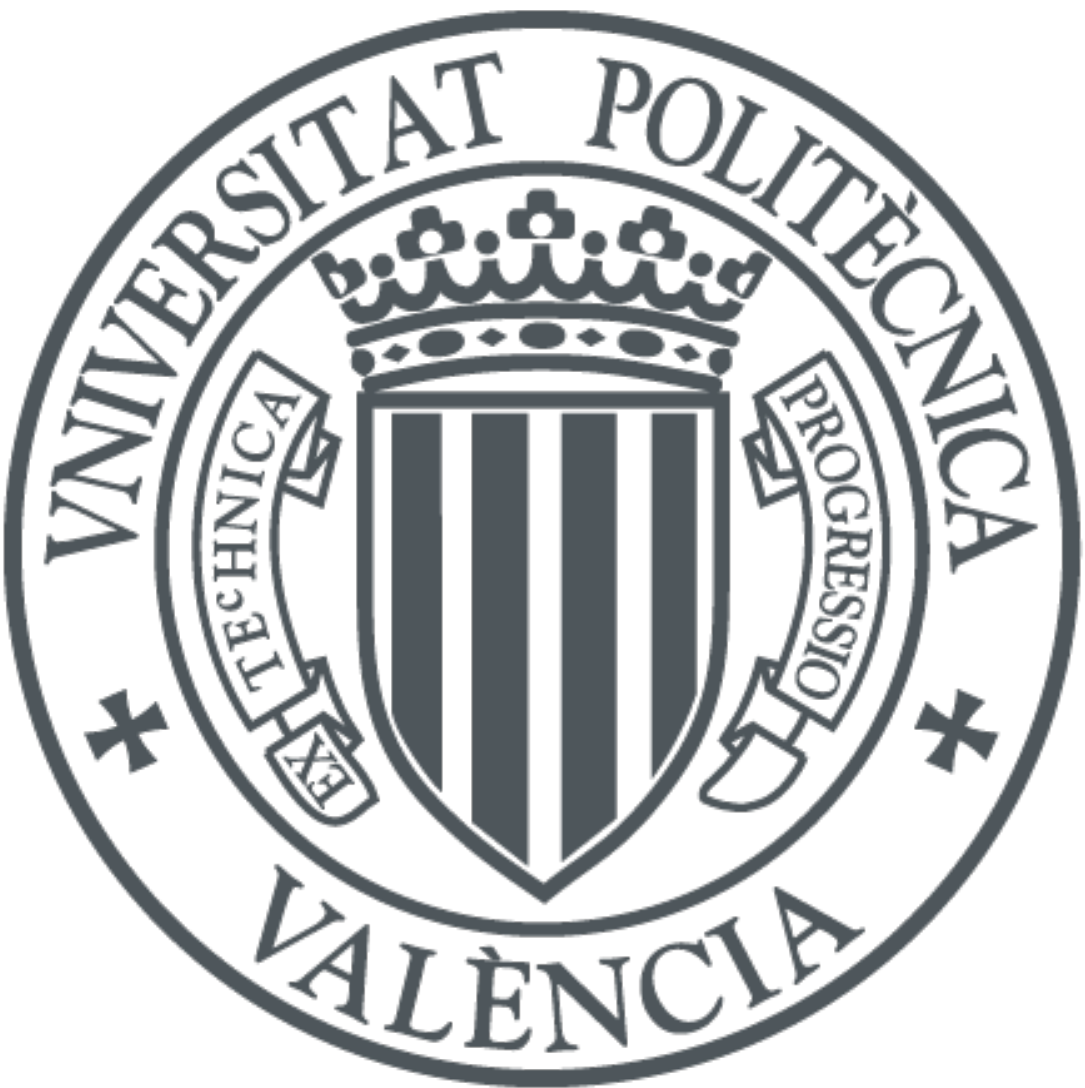

The final publication is available at

https://doi.org/10.1007/s11253-020-01851-5

Copyright Springer

Additional Information 


\section{Partial orders base on the CS decomposition}

Abstract: A new decomposition for square matrices was introduce by J. Benítez in [2]. In this paper, we will use this decomposition to investigate the minus, star, sharp and core partial orders in the setting of complex matrices.

Key words: Core-EP order, Minus partial order, Star partial order, Sharp partial order, Core partial order.

AMS subject classifications: 06A06, 15A09, 15A23.

\section{Introduction}

Let $\mathbb{C}^{m \times n}$ denote the set of all $m \times n$ complex matrices. Let $A^{*}, \mathcal{R}(A), \mathcal{N}(A)$ and $\operatorname{rk}(A)$ denote the conjugate transpose, column space, null space, and rank of $A \in \mathbb{C}^{m \times n}$, respectively. For $A \in \mathbb{C}^{m \times n}$, if $X \in \mathbb{C}^{n \times m}$ satisfies
(1) $A X A=A$,
(2) $X A X=X$
(3) $(A X)^{*}=A X$,
(4) $(X A)^{*}=X A$,

then $X$ is called a Moore-Penrose inverse of $A$. If such a matrix $X$ exists, then it is unique and denoted by $A^{\dagger}$. Let $I \subseteq\{1,2,3,4\}$. An element $B \in \mathbb{C}^{n \times m}$ is called an $I$ inverse of $A \in \mathbb{C}^{m \times n}$ if equalities $i \in I$ of $(\star)$ hold. The set of all $I$ inverses of $A$ will be denoted by $A^{I}$, the element $A$ is $I$ invertible when $A^{I} \neq \varnothing$.

Let $A \in \mathbb{C}^{n \times n}$. It can be easily proved that the set of elements $X \in \mathbb{C}^{n \times n}$ such that

$$
A X A=A, \quad X A X=X \quad \text { and } \quad A X=X A
$$

is empty or a singleton. If this set is a singleton, its unique element is called the group inverse of $A$ and denoted by $A^{\#}$.

The core inverse for a complex matrix was introduced by Baksalary and Trenkler [1]. Let $A \in \mathbb{C}^{n \times n}$. A matrix $X \in \mathbb{C}^{n \times n}$ is called a core inverse of $A$, if it satisfies $A X=P_{A}$ and $\mathcal{R}(X) \subseteq \mathcal{R}(A)$. Here $P_{A}$ denotes the orthogonal projector onto $\mathcal{R}(A)$. And if such a matrix exists, then it is unique (and denoted by $A^{\oplus}$ ). A square complex matrix $A$ is core invertible if and only if $\operatorname{rk}(A)=\operatorname{rk}\left(A^{2}\right)$ (see [1]), and let $\mathbb{C}_{n}^{C M}=\left\{A \in M_{n}(\mathbb{C}) \mid \operatorname{rk}(A)=\operatorname{rk}\left(A^{2}\right)\right\}$. The core partial order for complex matrices was also introduced in [1] and it is defined as follows: given $A \in \mathbb{C}_{n}^{C M}$ and $B \in M_{n}(\mathbb{C})$,

$$
A \stackrel{\oplus}{\leq} B \quad \Leftrightarrow \quad A^{\oplus} A=A^{\oplus} B \quad \text { and } \quad A A^{\oplus}=B A^{\oplus} .
$$

In [1, Theorem 6], it is proved that the core partial order is a matrix partial order. Baksalary and Trenkler [1] gave several characterizations and various relationships between the matrix core partial order and other matrix partial orders by using the decomposition of Hartwig and Spindelböck [8]. Let us recall some other well known partial orders in $\mathbb{C}^{n \times n}$. For $A, B \in \mathbb{C}^{n \times n}$,

- The star partial order $A \stackrel{*}{\leq} B: A^{*} A=A^{*} B$ and $A A^{*}=B A^{*}[6]$; 
- The minus partial order $A \leq B: A^{-} A=A^{-} B$ and $A A^{-}=B A^{-}[7]$, where $A^{-}$ denotes any inner inverse of $A$;

- The sharp partial order $A \stackrel{\#}{\leq} A: A^{\#} A=A^{\#} B$ and $A A^{\#}=B A^{\#}[13]$.

In addition, $\mathbf{1}_{n}$ and $\mathbf{0}_{n}$ will denote the $n \times 1$ column vectors all of whose components are 1 and 0 , respectively. $0_{m \times n}$ (abbr. 0) denotes the zero matrix of size $m \times n$. If $\mathcal{S}$ is a subspace of $\mathbb{C}^{n}$, then $P_{\mathcal{S}}$ stands for the orthogonal projector onto the subspace $S$. A matrix $A \in \mathbb{C}^{n \times n}$ is called an $E P$ matrix if $\mathcal{R}(A)=\mathcal{R}\left(A^{*}\right)$ and $A$ is unitary if $A A^{*}=I_{n}$, where $I_{n}$ denotes the identity matrix of size $n$.

\section{$2 \quad$ Preliminaries}

A related decomposition of the matrix decomposition of Hartwig and Spindelböck [8] was given in [2, Theorem 2.1] by Benítez. In [3] it can be found a simpler proof of this decomposition. Let us start this section with the concept of principal angles.

Definition 2.1. [19] Let $\mathcal{S}_{1}$ and $\mathcal{S}_{2}$ be two nontrivial subspaces of $\mathbb{C}^{n}$. We define the principal angles $\theta_{1}, \ldots, \theta_{r} \in[0, \pi / 2]$ between $\mathcal{S}_{1}$ and $\mathcal{S}_{2}$ by

$$
\cos \theta_{i}=\sigma_{i}\left(P_{\mathcal{S}_{1}} P_{\mathcal{S}_{2}}\right),
$$

for $i=1, \ldots, r$, where $r=\min \left\{\operatorname{dim} \mathcal{S}_{1}\right.$, $\left.\operatorname{dim} \mathcal{S}_{2}\right\}$. The real numbers $\sigma_{i}\left(P_{\mathcal{S}_{1}} P_{\mathcal{S}_{2}}\right) \geq 0$ are the singular values of $P_{\mathcal{S}_{1}} P_{\mathcal{S}_{2}}$.

Lemma 2.2. $\left[2\right.$, Theorem 2.1] Let $A \in \mathbb{C}^{n \times n}, r=\operatorname{rk}(A)$, and let $\theta_{1}, \ldots, \theta_{p}$ be the principal angles between $\mathcal{R}(A)$ and $\mathcal{R}\left(A^{*}\right)$ belonging to $] 0, \pi / 2[$. Denote by $x$ and $y$ the multiplicities of the angles 0 and $\pi / 2$ as a canonical angle between $\mathcal{R}(A)$ and $\mathcal{R}\left(A^{*}\right)$, respectively. There exists a unitary matrix $Y \in \mathbb{C}^{n \times n}$ such that

$$
A=Y\left[\begin{array}{cc}
M C & M S \\
0 & 0
\end{array}\right] Y^{*}
$$

where $M \in \mathbb{C}^{r \times r}$ is nonsingular,

$$
\begin{gathered}
C=\operatorname{diag}\left(\mathbf{0}_{y}, \cos \theta_{1}, \ldots, \cos \theta_{p}, \mathbf{1}_{x}\right), \\
S=\left[\begin{array}{cc}
\operatorname{diag}\left(\mathbf{1}_{y}, \sin \theta_{1}, \ldots, \sin \theta_{p}\right) & 0_{p+y, n-(r+p+y)} \\
0_{x, p+y} & 0_{x, n-(r+p+y)}
\end{array}\right],
\end{gathered}
$$

and $r=y+p+x$. Furthermore, $x$ and $y+n-r$ are the multiplicities of the singular values 1 and 0 in $P_{\mathcal{R}(A)} P_{\mathcal{R}\left(A^{*}\right)}$, respectively.

In this decomposition, one has $C^{2}+S S^{*}=I_{r}$. One has also

$$
A^{\dagger}=Y\left[\begin{array}{cc}
C M^{-1} & 0 \\
S^{*} M^{-1} & 0
\end{array}\right] Y^{*}, \quad A^{\#}=Y\left[\begin{array}{cc}
C^{-1} M^{-1} & C^{-1} M^{-1} C^{-1} S \\
0 & 0
\end{array}\right] Y^{*} .
$$

Recall that $A^{\dagger}$ always exists. We have that $A^{\#}$ exists if and only if $C$ is nonsingular [2, Theorem 3.7]. In this case, we have

$$
A^{\boxplus}=A^{\#} A A^{\dagger}=Y\left[\begin{array}{cc}
C^{-1} M^{-1} & 0 \\
0 & 0
\end{array}\right] Y^{*},
$$




$$
A A^{\boxplus}=Y\left[\begin{array}{cc}
I_{r} & 0 \\
0 & 0
\end{array}\right] Y^{*} \quad \text { and } \quad A^{\oplus} A=Y\left[\begin{array}{cc}
I_{r} & C^{-1} S \\
0 & 0
\end{array}\right] Y^{*} .
$$

We have

$$
A A^{\oplus}-A^{\oplus} A=Y\left[\begin{array}{cc}
I_{r} & 0 \\
0 & 0
\end{array}\right] Y^{*}-Y\left[\begin{array}{cc}
I_{r} & C^{-1} S \\
0 & 0
\end{array}\right] Y^{*}=Y\left[\begin{array}{cc}
0 & C^{-1} S \\
0 & 0
\end{array}\right] Y^{*}
$$

in view of (2.2). Now,

$$
\operatorname{det}\left(A A^{\oplus}-A^{\oplus} A\right)=\operatorname{det}\left(Y\left[\begin{array}{cc}
0 & C^{-1} S \\
0 & 0
\end{array}\right] Y^{*}\right)=0 .
$$

Thus, $A A^{\oplus}-A^{\oplus} A$ is always singular and $\operatorname{rk}\left(A A^{\oplus}-A^{\oplus} A\right)=\operatorname{rk}\left(C^{-1} S\right)=\operatorname{rk}(S)<n$. From (2.3), we have that $A$ is an EP matrix if and only if $S=0$, that is all the canonical angles between $\mathcal{R}(A)$ and $\mathcal{R}\left(A^{*}\right)$ are 0 . This result also can be found in [2, Theorem 3.7].

Proposition 2.3. If $A \in \mathbb{C}^{n \times n}$ is core invertible and $A$ has the form (2.1), then $A A^{\boxplus}-$ $A^{\boxplus} A$ is always singular with $\operatorname{rk}\left(A A^{\boxplus}-A^{\boxplus} A\right)=\operatorname{rk}(S)<n$.

In [21, Theorem 3.1], the authors proved the following lemma for an element in a ring with involution.

Lemma 2.4. Let $A \in \mathbb{C}^{n \times n}$. Then $A$ is core invertible with $A^{\boxplus}=X$ if and only if $(A X)^{*}=A X, X A^{2}=A$ and $A X^{2}=X$.

Proposition 2.5. Let $A, B, U \in \mathbb{C}^{n \times n}$ with $A=U B U^{*}$, where $B$ is core invertible and $U$ is unitary. Then $A$ is core invertible. In this case, one has $A^{\boxplus}=U B^{\boxplus} U^{*}$.

Proof. Let $X=U B^{\oplus} U^{*}$, we have

$$
\begin{aligned}
A X & =A U B^{\oplus} U^{*}=U B U^{*} U B^{\oplus} U^{*}=U B B^{\oplus} U^{*} \text { is Hermitian, } \\
X A^{2} & =U B^{\oplus} U^{*}\left(U B U^{*}\right)^{2}=U B^{\oplus}(B)^{2} U^{*}=U B U^{*}=A, \\
A X^{2} & =U A U^{*}\left(U B U^{*}\right)^{2}=U B\left(B^{\oplus}\right)^{2} U^{*}=U B^{\oplus} U^{*}=X .
\end{aligned}
$$

Thus, $A^{\oplus}=U B^{\oplus} U^{*}$ in view of Lemma 2.4.

Recently, Wang introduced a new decomposition for square matrices, named Core-EP decomposition in [18, Theorem 2.1].

Lemma 2.6. Let $A \in \mathbb{C}^{n \times n}$ with $\operatorname{ind}(A)=k$. Then $A$ can be written as

$$
A=A_{1}+A_{2}
$$

in which

(1) $A_{1} \in \mathbb{C}_{n}^{C M}$;

(2) $A_{2}^{k}=0$;

(3) $A_{1}^{*} A_{2}=A_{2} A_{1}=0$. 
We call the equality (2.4) the Core-EP decomposition of $A$.

Definition 2.7. [11, Definition 3.1] Let $A \in \mathbb{C}^{n \times n}$ with $\operatorname{ind}(A)=k$. A matrix $X \in \mathbb{C}^{n \times n}$ is the core-EP inverse of $A$ (is unique and denoted by $A^{\oplus}$ ) if $X$ is an outer inverse of $A$ and satisfies

$$
\mathcal{R}(X)=\mathcal{R}\left(X^{*}\right)=\mathcal{R}\left(A^{k}\right) .
$$

Lemma 2.8. [11, Lemma 3.3] Let $A \in \mathbb{C}^{n \times n}$ with ind $(A)=k$. Then $X \in \mathbb{C}^{n \times n}$ is the core-EP inverse of $A$ if and only if

$$
X A^{k+1}=A^{k}, \quad X A X=X, \quad(A X)^{*}=A X \text { and } \mathcal{R}(X) \subseteq \mathcal{R}\left(A^{k}\right) .
$$

\section{A matrix decomposition related the CS decomposition and the core-EP decomposition}

Theorem 3.1. Let $A \in \mathbb{C}^{n \times n}$ with $\operatorname{ind}(A)=k$ and $r=\operatorname{rk}(A)$. There exists a unitary matrix $U \in \mathbb{C}^{n \times n}$ such that

$$
A=U\left[\begin{array}{cc}
M C & M S \\
0 & D_{4}
\end{array}\right] U^{*}
$$

where $M$ and $C$ are both nonsingular, $D_{4}$ is nilpotent, $C^{2}+S S^{*}=I_{r}$ and matrices $C$ and $S$ have the form after equality (2.1).

Proof. From Lemma 2.6, we have

$$
A=A_{1}+A_{2},
$$

in which $A_{1} \in \mathbb{C}_{n}^{C M}, A_{2}^{k}=0$ and $A_{1}^{*} A_{2}=A_{2} A_{1}=0$. Now, applying Lemma 2.2 to $A_{1}$, there exists a unitary matrix $U$ such that

$$
A_{1}=U\left[\begin{array}{cc}
M C & M S \\
0 & 0
\end{array}\right] U^{*}
$$

in which $M$ is nonsingular. We also have $C$ is nonsingular in view of $A_{1} \in \mathbb{C}_{n}^{C M}$ and [2, Theorem 3.7]. Let $A_{2}=U\left[\begin{array}{ll}D_{1} & D_{2} \\ D_{3} & D_{4}\end{array}\right] U^{*}$. And

$$
\begin{aligned}
& A_{1}^{*} A_{2}=U\left[\begin{array}{cc}
C M^{*} & 0 \\
S^{*} M^{*} & 0
\end{array}\right]\left[\begin{array}{cc}
D_{1} & D_{2} \\
D_{3} & D_{4}
\end{array}\right] U^{*}=U\left[\begin{array}{cc}
C M^{*} D_{1} & C M^{*} D_{2} \\
S^{*} M^{*} D_{1} & S^{*} M^{*} D_{2}
\end{array}\right] U^{*} \\
& A_{2} A_{1}=U\left[\begin{array}{cc}
D_{1} & D_{2} \\
D_{3} & D_{4}
\end{array}\right]\left[\begin{array}{cc}
M C & M S \\
0 & 0
\end{array}\right] U^{*}=U\left[\begin{array}{cc}
D_{1} M C & D_{1} M S \\
D_{3} M C & D_{3} M S
\end{array}\right] U^{*}
\end{aligned}
$$

From (3.2) and (3.3) and $A_{1}^{*} A_{2}=A_{2} A_{1}=0$ we get

$$
C M^{*} D_{1}=0 ; \quad C M^{*} D_{2}=0 ; \quad D_{3} M C=0 .
$$

The nonsingularity of $C$ and $M$ implies that $D_{1}, D_{2}$ and $D_{3}$ are zero matrices. Thus

$$
A=A_{1}+A_{2}=U\left[\begin{array}{cc}
M C & M S \\
0 & D_{4}
\end{array}\right] U^{*} \text {. }
$$

The equality $A_{2}^{k}=0$ implies that $D_{4}$ is nilpotent. 
Note that the decomposition in Theorem 3.1 has the same form as Schur form, but the decomposition seems easier to handle. Have in mind that $M$ and $C$ are both nonsingular, $C$ is real and diagonal, $D_{4}$ is nilpotent and $C^{2}+S S^{*}=I$ by Theorem 3.1.

Since $C$ is nonsingular, we have $A_{1}^{\#}=U\left[\begin{array}{c}C^{-1} M^{-1} C^{-1} M_{0}^{-1} C^{-1} S \\ 0\end{array} U^{*}\right.$ by [2, Theorem 3.7]. It is evident that $A_{1}^{\oplus}=U\left[\begin{array}{cc}C^{-1} M^{-1} & 0 \\ 0 & 0\end{array}\right] U^{*}$. From [18, Theorem 3.2], we have $A^{\oplus}=A_{1}^{\oplus}=$ $U\left[\begin{array}{cc}C^{-1} M^{-1} & 0 \\ 0 & 0\end{array}\right] U^{*}$.

In the following theorem, we will use the matrix decomposition in Theorem 3.1 to investigate the core-EP order, which was introduced by Wang in [18], defined as: for matrices $A, B \in \mathbb{C}^{n \times n}$

$$
A \stackrel{\oplus}{\leq} B \quad \Leftrightarrow \quad A^{\oplus} A=A^{\oplus} B \text { and } A A^{\oplus}=B A^{\oplus} .
$$

Theorem 3.2. Let $A, B \in \mathbb{C}^{n \times n}$. Assume that $A$ has the form (3.1). If $A$ is core-EP invertible, then $A \stackrel{\oplus}{\leq} B$ if and only if $B-A$ can be written as

$$
B-A=U\left[\begin{array}{ll}
0 & 0 \\
0 & E
\end{array}\right] U^{*}, \quad E \in \mathbb{C}^{(n-r) \times(n-r)} .
$$

Proof. Let $B-A=U\left[\begin{array}{ll}B_{1} & B_{2} \\ B_{3} & B_{4}\end{array}\right] U^{*}$, where $B_{1} \in \mathbb{C}^{r \times r}$ and suppose $A \stackrel{\oplus}{\leq} B$, then

$$
\begin{aligned}
& A^{\oplus}(B-A)=U\left[\begin{array}{cc}
C^{-1} M^{-1} B_{1} & C^{-1} M^{-1} B_{2} \\
0 & 0
\end{array}\right] U^{*} ; \\
& (B-A) A^{\oplus}=U\left[\begin{array}{ll}
B_{1} C^{-1} M^{-1} & 0 \\
B_{3} C^{-1} M^{-1} & 0
\end{array}\right] U^{*} .
\end{aligned}
$$

From (3.4), (3.5), $A^{\oplus} A=A^{\oplus} B$ and $A A^{\oplus}=B A^{\oplus}$, we get

$$
C^{-1} M^{-1} B_{1}=0 ; \quad C^{-1} M^{-1} B_{2}=0 ; \quad B_{3} C^{-1} M^{-1}=0 .
$$

From (3.6) and the nonsingularity of $C$ and $M$ it follows that $B_{1}, B_{2}, B_{3}$ are zero matrices.

To prove the opposite implication, it is easy to check that $(B-A) A^{\oplus}=A^{\oplus}(B-A)=0$, that is $A \stackrel{\oplus}{\leq} B$.

\section{Core, star, group and minus partial order}

In this section, we consider the relationships between the core partial order and other partial orders by using Lemma 2.2 for square matrices. Let $A \in \mathbb{C}^{n \times n}$. Recall that the left star partial order $A * \leq B$ in $\mathbb{C}^{n \times n}$ is defined by $A^{*} A=A^{*} B$ and $\mathcal{R}(A) \subseteq \mathcal{R}(B)$. The right sharp partial order $A \leq_{\#} B$ is defined as: $A A^{\#}=B A^{\#}$ and $\mathcal{R}\left(A^{*}\right) \subseteq \mathcal{R}\left(B^{*}\right)$. Let us begin with some lemmas will be useful in the sequel.

Lemma 4.1. [13, Lemma 2.2] Let $A \in \mathbb{C}^{n \times n}$ be group invertible. Then $A \stackrel{\#}{\leq} B$ if and only if $A^{2}=A B=B A$. 
Lemma 4.2. $\left[22\right.$, Theorem 3.2] Let $A, B \in \mathbb{C}^{n \times n}$ be two core invertible matrices. Then $A \stackrel{\oplus}{\leq} B$ if and only if $A * \leq B$ and $B^{\oplus} A A^{\oplus}=A^{\oplus}$

An equivalent form of the minus partial order is the following statement: for the complex case can be found in $[4,12]$ and for the ring case can be found in [10].

Lemma 4.3. Let $A, B \in \mathbb{C}^{n \times n}$. Then the following are equivalent:

(1) $B \leq A$

(2) There exists $A^{-} \in A\{1\}$ such that $B=A A^{-} B=B A^{-} A=B A^{-} B$;

(3) $B=A A^{-} B=B A^{-} A=B A^{-} B$ for all $A^{-} \in A\{1\}$.

The following lemma was proved in the more general setting of rings with an involution in [16, Theorem 4.10].

Lemma 4.4. Let $A, B \in \mathbb{C}^{n \times n}$. If $A, B$ are both core invertible and $B \overline{\leq} A$, then $B \stackrel{\oplus}{\leq} A$ if and only if $A^{\oplus B} A^{\oplus}=B^{\oplus}$.

Theorem 4.5. Let $A, B \in \mathbb{C}^{n \times n}$. Assume that $A$ has the form (2.1). If $A$ is core invertible, then the following are equivalent:

(1) $A \stackrel{\oplus}{\leq} B$;

(2) $B-A$ can be written as

$$
B-A=Y\left[\begin{array}{cc}
0 & 0 \\
0 & B_{4}
\end{array}\right] Y^{*}, \quad B_{4} \in \mathbb{C}^{(n-r) \times(n-r)}
$$

(3) $P_{A}(B-A)=0$ and $(B-A) P_{A}=0$, where $P_{A}=A A^{\oplus}$;

(4) $B=A+\left(I_{n}-A A^{\boxplus}\right) X\left(I_{n}-A A^{\boxplus}\right)$ for some matrix $X \in \mathbb{C}^{n \times n}$.

Proof. (1) $\Leftrightarrow(2)$. Since $A$ is core invertible and the core invertibility is equivalent to the group invertibility, matrix $C$ is nonsingular. Let $B=Y\left[\begin{array}{ll}B_{1} & B_{2} \\ B_{3} & B_{4}\end{array}\right] Y^{*}$. If $A \stackrel{\oplus}{\leq} B$, then $A A^{\oplus}=B A^{\oplus}, A^{\oplus} B=A^{\oplus} A$. Thus $A A^{\oplus} B=A=B A^{\oplus} A$ by $A A^{\oplus} A=A$.

$$
\begin{aligned}
& A A^{\oplus} B=Y\left[\begin{array}{cc}
I_{r} & 0 \\
0 & 0
\end{array}\right]\left[\begin{array}{cc}
B_{1} & B_{2} \\
B_{3} & B_{4}
\end{array}\right] Y^{*}=Y\left[\begin{array}{cc}
B_{1} & B_{2} \\
0 & 0
\end{array}\right] Y^{*} ; \\
& B A^{\oplus} A=Y\left[\begin{array}{ll}
B_{1} & B_{2} \\
B_{3} & B_{4}
\end{array}\right]\left[\begin{array}{cc}
I_{r} & C^{-1} S \\
0 & 0
\end{array}\right] Y^{*}=Y\left[\begin{array}{cc}
B_{1} & B_{1} C^{-1} S \\
B_{3} & B_{3} C^{-1} S
\end{array}\right] Y^{*} .
\end{aligned}
$$

From (4.2) and (4.3) we get $B_{1}=M C, B_{2}=M S$ and $B_{3}=0$. Thus

$$
B=Y\left[\begin{array}{cc}
M C & M S \\
0 & B_{4}
\end{array}\right] Y^{*}=Y\left[\begin{array}{cc}
M C & M S \\
0 & 0
\end{array}\right] Y^{*}+Y\left[\begin{array}{cc}
0 & 0 \\
0 & B_{4}
\end{array}\right] Y^{*}=A+Y\left[\begin{array}{cc}
0 & 0 \\
0 & B_{4}
\end{array}\right] Y^{*}
$$


That is (4.1). Conversely, if we have (4.1), it is easy to check that $A A^{\boxplus} B=A$, which is equivalent to $A^{\oplus} A=A^{\oplus} B$. And we have $A A^{\oplus}=B A^{\oplus}$ in a similar way.

$(2) \Rightarrow(3)$. Since we have $A^{\boxplus}=A^{\#} A A^{\dagger}=Y\left[\begin{array}{cc}C^{-1} M^{-1} & 0 \\ 0 & 0\end{array}\right] Y^{*}$, so $A A^{\oplus}=Y\left[\begin{array}{cc}I_{r} & 0 \\ 0 & 0\end{array}\right] Y^{*}$. It is easy to check that $P_{A}(B-A)=0$ and $(B-A) P_{A}=0$.

$(3) \Rightarrow(2)$. Let $B-A=Y\left[\begin{array}{ll}X_{1} & X_{2} \\ X_{3} & X_{4}\end{array}\right] Y^{*}$, where $X_{1} \in \mathbb{C}^{r \times r}$. The hypothesis $P_{A}(B-A)=$ 0 implies that $X_{1}$ and $X_{2}$ are zero matrices and $(B-A) P_{A}=0$ implies that $X_{3}=0$. Thus we have the form in (4.1).

$(2) \Rightarrow(4)$. Note that (4.1) can be written as

$$
\begin{aligned}
B-A & =Y\left[\begin{array}{cc}
0 & 0 \\
0 & I_{n-r}
\end{array}\right]\left[\begin{array}{cc}
0 & 0 \\
0 & B_{4}
\end{array}\right]\left[\begin{array}{cc}
0 & 0 \\
0 & I_{n-r}
\end{array}\right] Y^{*} \\
& =Y\left\{\left(\left[\begin{array}{cc}
I_{r} & 0 \\
0 & I_{n-r}
\end{array}\right]-\left[\begin{array}{cc}
I_{r} & 0 \\
0 & 0
\end{array}\right]\right)\left[\begin{array}{cc}
0 & 0 \\
0 & B_{4}
\end{array}\right]\left(\left[\begin{array}{cc}
I_{r} & 0 \\
0 & I_{n-r}
\end{array}\right]-\left[\begin{array}{cc}
I_{r} & 0 \\
0 & 0
\end{array}\right]\right)\right\} Y^{*} .
\end{aligned}
$$

Therefore, $B-A=\left(I_{n}-P_{A}\right) X\left(I_{n}-P_{A}\right)$ for some matrix $X$. Let $Q=I_{n}-P_{A}$, we get that $Q A=0$ and $B-A=Q X Q$ for some matrix $X$.

$(4) \Rightarrow(3)$ is trivial.

Remark 4.6. When $\operatorname{ind}(A)=1$, from [11, Theorem 3.8] we have the core-EP inverse coincides with the core inverse. Thus, the equivalence between (1) and (2) in Theorem 4.5 also can be got by Theorem 3.2.

Theorem 4.7. Let $A, B \in \mathbb{C}^{n \times n}$. Assume that $A$ has the form (2.1). If $A$ is group invertible, then the following are equivalent:

(1) $A \stackrel{*}{\leq} B$

(2) $B-A$ can be written as

$$
B-A=Y\left[\begin{array}{cc}
0 & 0 \\
-B_{4} S^{*} C^{-1} & B_{4}
\end{array}\right] Y^{*}, \quad B_{4} \in \mathbb{C}^{(n-r) \times(n-r)}
$$

(3) $A A^{\dagger}(B-A)=0$ and $(B-A) A A^{\dagger}=(B-A) Y\left[\begin{array}{cc}0 & 0 \\ -S^{*} C^{-1} & 0\end{array}\right] Y^{*}$;

(4) $B=A+\left(I_{n}-A A^{\dagger}\right) X\left(I_{n}-A A^{\dagger}\right)\left(I-A^{\#} A\right)^{*}$ for some matrix $X \in \mathbb{C}^{n \times n}$.

Proof. (1) $\Leftrightarrow(2)$. Since $A$ is group invertible, matrix $C$ is nonsingular. Let $B-A=$ $Y\left[\begin{array}{ll}B_{1} & B_{2} \\ B_{3} & B_{4}\end{array}\right] Y^{*}$, where $B_{1} \in \mathbb{C}^{r \times r}$. Suppose $A \stackrel{*}{\leq} B$. Hence $A^{*} A=A^{*} B$ and $A A^{*}=$ $B A^{*}$. We marked with $\star$, the entries that we are not interest in.

$$
\begin{aligned}
& A^{*}(B-A)=Y\left[\begin{array}{cc}
C M^{*} & 0 \\
S^{*} M^{*} & 0
\end{array}\right]\left[\begin{array}{cc}
B_{1} & B_{2} \\
B_{3} & B_{4}
\end{array}\right] Y^{*}=Y\left[\begin{array}{cc}
C M^{*} B_{1} & C M^{*} B_{2} \\
\star & \star
\end{array}\right] Y^{*} \\
& (B-A) A^{*}=Y\left[\begin{array}{ll}
B_{1} & B_{2} \\
B_{3} & B_{4}
\end{array}\right]\left[\begin{array}{cc}
C M^{*} & 0 \\
S^{*} M^{*} & 0
\end{array}\right] Y^{*}=Y\left[\begin{array}{cc}
\star & 0 \\
B_{3} C M^{*}+B_{4} S^{*} M^{*} & 0
\end{array}\right] Y^{*}
\end{aligned}
$$


From (4.5) we get $C M^{*} B_{1}=0$ and $C M^{*} B_{2}=0$. The nonsingularity of $C$ and $M$ implies that $B_{1}=0$ and $B_{2}=0$. From (4.6) we get $B_{3} C M^{*}+B_{4} S^{*} M^{*}=0$. The nonsingularity of $C$ and $M$ leads to $B_{3}=-B_{4} S^{*} C^{-1}$. Conversely, we have

$$
\begin{aligned}
& (B-A) A^{*}=Y\left[\begin{array}{cc}
0 & 0 \\
-B_{4} S^{*} C^{-1} & B_{4}
\end{array}\right]\left[\begin{array}{cc}
C M^{*} & 0 \\
S^{*} M^{*} & 0
\end{array}\right] Y^{*}=0 \\
& A^{*}(B-A)=Y\left[\begin{array}{cc}
C M^{*} & 0 \\
S^{*} M^{*} & 0
\end{array}\right]\left[\begin{array}{cc}
0 & 0 \\
-B_{4} S^{*} C^{-1} & B_{4}
\end{array}\right] Y^{*}=0 .
\end{aligned}
$$

From (4.7) and (4.8), we get $B A^{*}=A A^{*}$ and $A^{*} B=A^{*} A$. That is $A \stackrel{*}{\leq} B$.

$(2) \Rightarrow(3)$. Since we have $A^{\dagger}=Y\left[\begin{array}{cc}C M^{-1} & 0 \\ S^{*} M^{-1} & 0\end{array}\right] Y^{*}$, so $A A^{\dagger}=Y\left[\begin{array}{cc}I_{r} & 0 \\ 0 & 0\end{array}\right] Y^{*}$. It is easy to check that $A A^{\dagger}(B-A)=0$ and $(B-A) A A^{\dagger}=(B-A) Y\left[\begin{array}{cc}0 & 0 \\ -S^{*} C^{-1} & 0\end{array}\right] Y^{*}$.

$(3) \Rightarrow(2)$. Let $B-A=Y\left[\begin{array}{ll}X_{1} & X_{2} \\ X_{3} & X_{4}\end{array}\right] Y^{*}$, where $X_{1} \in \mathbb{C}^{r \times r}$. From $A A^{\dagger}(B-A)=0$ we get that $X_{1}$ and $X_{2}$ are zero matrices and $(B-A) A A^{\dagger}=(B-A) Y\left[\begin{array}{cc}0 & 0 \\ -S^{*} C^{-1} & 0\end{array}\right] Y^{*}$ implies $X_{3}=-X_{4} S^{*} C^{-1}$. Thus we have the form in (4.4).

$(2) \Rightarrow(4)$. Note that (4.4) can be written as

$$
\begin{aligned}
B-A & =Y\left[\begin{array}{cc}
0 & 0 \\
0 & B_{4}
\end{array}\right]\left[\begin{array}{cc}
0 & 0 \\
-S^{*} C^{-1} & I_{n-r}
\end{array}\right] Y^{*} \\
& =Y\left\{\left[\begin{array}{cc}
0 & 0 \\
0 & B_{4}
\end{array}\right]\left(\left[\begin{array}{cc}
I_{r} & 0 \\
0 & I_{n-r}
\end{array}\right]-\left[\begin{array}{cc}
I_{r} & C^{-1} S \\
0 & 0
\end{array}\right]\right)^{*}\right\} Y^{*} \\
& =Y\left\{\left[\begin{array}{cc}
0 & 0 \\
0 & B_{4}
\end{array}\right]\left(I-A^{\#} A\right)^{*}\right\} Y^{*}
\end{aligned}
$$

Therefore, $B-A=\left(I_{n}-A A^{\dagger}\right) X\left(I_{n}-A A^{\dagger}\right)\left(I-A^{\#} A\right)^{*}$ for some matrix $X \in \mathbb{C}^{n \times n}$.

(4) $\Rightarrow(1)$. Since $A^{*}\left(I_{n}-A A^{\dagger}\right)=A^{*}\left(I_{n}-A A^{\dagger}\right)^{*}=0$ we obtain $A^{*}(B-A)=0$. Since $\left(I_{n}-A^{\#} A\right)^{*} A^{*}=\left[A\left(I_{n}-A^{\#} A\right)\right]^{*}=0$, we get $(B-A) A^{*}=0$.

Theorem 4.8. Let $A, B \in \mathbb{C}^{n \times n}$. Assume that $A$ has the form (2.1). If $A$ is group invertible, then the following are equivalent:

(1) $A \stackrel{\#}{\leq} B$

(2) $B-A$ can be written as

$$
B-A=Y\left[\begin{array}{cc}
0 & -C^{-1} S B_{4} \\
0 & B_{4}
\end{array}\right] Y^{*}, \quad B_{4} \in \mathbb{C}^{(n-r) \times(n-r)}
$$

(3) $A A^{\#}(B-A)=(B-A) A A^{\#}=0$;

(4) There exists a projection $Q$ such that $Q A=0$ and $B-A=\left(I-A^{\#} A\right) Q X Q$ for some matrix $X \in \mathbb{C}^{n \times n}$.

In this case, $A \stackrel{\#}{\leq} B$ if and only if exists $X \in \mathbb{C}^{n \times n}$ such that $B=A+\left(I_{n}-A^{\#} A\right)\left(I_{n}-\right.$ $\left.A A^{\dagger}\right) X\left(I_{n}-A A^{\dagger}\right)$. 
Proof. (1) $\Rightarrow(2)$. Since $A$ is group invertible, we get that $C$ is nonsingular. Let $B-A=$ $Y\left[\begin{array}{ll}B_{1} & B_{2} \\ B_{3} & B_{4}\end{array}\right] Y^{*}$, where $B_{1} \in \mathbb{C}^{r \times r}$. Since $A \stackrel{\#}{\leq} B$, then $A B=A^{2}=B A$ by Lemma 4.1, i.e., $A(B-A)=(B-A) A=0$. We marked with $\star$, the entries that we are not interest in.

$$
0=(B-A) A=Y\left[\begin{array}{ll}
B_{1} & B_{2} \\
B_{3} & B_{4}
\end{array}\right]\left[\begin{array}{cc}
M C & M S \\
0 & 0
\end{array}\right] Y^{*}=Y\left[\begin{array}{cc}
B_{1} M C & B_{1} M S \\
B_{3} M C & B_{3} M S
\end{array}\right] Y^{*} .
$$

The nonsingularity of $M$ and $C$ imply that $B_{1}$ and $B_{3}$ are zero matrices.

$$
0=A(B-A)=Y\left[\begin{array}{cc}
M C & M S \\
0 & 0
\end{array}\right]\left[\begin{array}{ll}
0 & B_{2} \\
0 & B_{4}
\end{array}\right] Y^{*}=Y\left[\begin{array}{cc}
0 & M\left(C B_{2}+S B_{4}\right) \\
0 & 0
\end{array}\right]
$$

The nonsingularity of $M$ and $C$ imply $B_{2}=-C^{-1} S B_{4}$, i.e., we have obtained (4.9).

$(2) \Rightarrow(1)$. Conversely, we have

$$
\begin{aligned}
& (B-A) A=Y\left[\begin{array}{cc}
0 & -C^{-1} S B_{4} \\
0 & B_{4}
\end{array}\right]\left[\begin{array}{cc}
M C & M S \\
0 & 0
\end{array}\right] Y^{*}=0 \\
& A(B-A)=Y\left[\begin{array}{cc}
M C & M S \\
0 & 0
\end{array}\right]\left[\begin{array}{cc}
0 & -C^{-1} S B_{4} \\
0 & B_{4}
\end{array}\right] Y^{*}=0
\end{aligned}
$$

From (4.10) and (4.11), we get $A^{2}=A B=B A$. That is $A \stackrel{\#}{\leq} B$.

$(1) \Rightarrow(3)$. From $A B=A^{2}$, we get $A^{\#} A B=A$, and now $A A^{\#}(B-A)=A A^{\#} B-A=$ 0 . The equality $(B-A) A A^{\#}=0$ is obtained in a similar way.

$(3) \Rightarrow(1)$. Since $A A^{\#}(B-A)=0$ and $(B-A) A A^{\#}=0$ are equivalent to $A A^{\#} B=A$ and $B A A^{\#}=A$, respectively, we can get $A^{\#} B=A^{\#} A$ and $A A^{\#}=B A^{\#}$ by multiplying $A^{\#}$ on the left side of $A A^{\#} B=A$ and multiplying $A^{\#}$ on the right side of $B A A^{\#}=A$. That is $A \stackrel{\#}{\leq} B$ by the definition of the sharp star partial order.

$(2) \Rightarrow(4)$. Note that (4.9) can be written as

$$
\begin{aligned}
B-A & =Y\left[\begin{array}{cc}
0 & -C^{-1} S \\
0 & I_{n-r}
\end{array}\right]\left[\begin{array}{cc}
0 & 0 \\
0 & B_{4}
\end{array}\right] Y^{*} \\
& =Y\left\{\left(\left[\begin{array}{cc}
I_{r} & 0 \\
0 & I_{n-r}
\end{array}\right]-\left[\begin{array}{cc}
I_{r} & C^{-1} S \\
0 & 0
\end{array}\right]\right)\left[\begin{array}{cc}
0 & 0 \\
0 & B_{4}
\end{array}\right]\right\} Y^{*} .
\end{aligned}
$$

Therefore, $B-A=\left(I_{n}-A^{\#} A\right)\left(I_{n}-A A^{\dagger}\right) X\left(I_{n}-A A^{\dagger}\right)$ for some matrix $X \in \mathbb{C}^{n \times n}$. Let $Q=I_{n}-A A^{\dagger}$, we get that $Q$ is a projection such that $Q A=0$.

$(4) \Rightarrow(1)$. Multiplying by $A$ on the left side of $B-A=\left(I-A^{\#} A\right) Q X Q$, we obtain $A^{2}=A B$, and multiplying by $A$ on the right side of $B-A=\left(I-A^{\#} A\right) Q X Q$, we obtain $A^{2}=B A$. Thus $A \stackrel{\#}{\leq} B$ by lemma 4.1 .

Let $A, B \in \mathbb{C}^{n \times n}$ and let $A$ be a group invertible matrix. If $A$ is an EP matrix, then $A(A-B)=0 \Leftrightarrow \mathcal{R}(A-B) \subseteq \mathcal{N}(A) \Leftrightarrow \mathcal{R}(A-B) \subseteq \mathcal{N}\left(A^{*}\right) \Leftrightarrow A^{*}(A-B)=0$ and $(A-B) A=0 \Leftrightarrow A^{*}\left(A^{*}-B^{*}\right)=0 \Leftrightarrow \mathcal{R}\left(A^{*}-B^{*}\right) \subseteq \mathcal{N}\left(A^{*}\right) \Leftrightarrow \mathcal{R}\left(A^{*}-B^{*}\right) \subseteq \mathcal{N}(A) \Leftrightarrow$ $A\left(A^{*}-B^{*}\right)=0 \Leftrightarrow(A-B) A^{*}=0$, which proves that if $A$ is an EP matrix, then $A \stackrel{\#}{\leq} B$ if and only if $A \stackrel{*}{\leq} B$. 
Theorem 4.9. Let $A, B \in \mathbb{C}^{n \times n}$ be core invertible. Then $A \stackrel{\oplus}{\leq} B$ if and only if $A * \leq B$ and $\mathcal{R}(A) \subseteq \mathcal{N}\left(B^{\oplus}-A^{\oplus}\right)$.

Proof. By Lemma 4.2, it is enough to prove that $B^{\oplus} A A^{\oplus}=A^{\oplus}$ if and only if $\mathcal{R}(A) \subseteq$ $\mathcal{N}\left(B^{\oplus}-A^{\oplus}\right)$.

If $B^{\oplus} A A^{\oplus}=A^{\oplus}$, then $\left(B^{\oplus}-A^{\oplus}\right)\left(I_{n}-A A^{\oplus}\right)=B^{\oplus}-A^{\oplus}$, and thus, exists $X \in$ $\mathbb{C}^{n \times n}$ such that $B^{\oplus}-A^{\oplus}=X\left(I_{n}-A A^{\oplus}\right)$. And $B^{\oplus}-A^{\oplus}=X\left(I_{n}-A A^{\oplus}\right)$ implies $\left(B^{\boxplus}-A^{\oplus}\right)^{*}=\left(I_{n}-A A^{\boxplus}\right) X^{*}$, hence $\mathcal{R}\left[\left(B^{\oplus}-A^{\boxplus}\right)^{*}\right] \subseteq \mathcal{R}\left(I_{n}-A A^{\oplus}\right)$. But $\mathcal{R}\left[\left(B^{\oplus}-\right.\right.$ $\left.\left.A^{\oplus}\right)^{*}\right]=\left[\mathcal{N}\left(B^{\oplus}-A^{\oplus}\right)\right]^{\perp}$ and by using that $A A^{\oplus}$ is the orthogonal projector onto $\mathcal{R}(A)$, we have $\mathcal{R}\left(I_{n}-A A^{\oplus}\right)=\mathcal{R}\left(A A^{\oplus}\right)^{\perp}=\mathcal{R}(A)^{\perp}$. Therefore, $\left[\mathcal{N}\left(B^{\oplus}-A^{\oplus}\right)\right]^{\perp} \subseteq \mathcal{R}(A)^{\perp}$, hence $\mathcal{R}(A) \subseteq \mathcal{N}\left(B^{\boxplus}-A^{\boxplus}\right)$.

Conversely, if $\mathcal{R}(A) \subseteq \mathcal{N}\left(B^{\oplus}-A^{\oplus}\right)$, then $\mathcal{R}\left[\left(B^{\oplus}-A^{\oplus}\right)^{*}\right]=\left[\mathcal{N}\left(B^{\oplus}-A^{\boxplus}\right)\right]^{\perp} \subseteq$ $[\mathcal{R}(A)]^{\perp}=\mathcal{R}\left(A A^{\oplus}\right)^{\perp}=\mathcal{R}\left(I_{n}-A A^{\oplus}\right)$, hence $B^{\oplus}-A^{\oplus}=X^{\prime}\left(I_{n}-A A^{\oplus}\right)$ for some matrix $X^{\prime} \in \mathbb{C}^{n \times n}$. Therefore $B^{\oplus} A A^{\oplus}=\left[A^{\oplus}+X^{\prime}\left(I_{n}-A A^{\oplus}\right)\right] A A^{\oplus}=A^{\oplus} A A^{\oplus}=A^{\oplus}$.

Let $A, B \in \mathbb{C}^{n \times n}$. To study a partial order between $A$ and $B$, we have two ways. One is to use the CS decomposition of $A$; another is to use the CS decomposition of $B$.

Theorem 4.10. Let $A, B \in \mathbb{C}^{n \times n}$ be group invertible. Assume that $A$ has the form (2.1). Then $B \leq A$ if and only if $B$ can be written as

$$
B=Y\left[\begin{array}{cc}
B_{1} & B_{1} C^{-1} S \\
0 & 0
\end{array}\right] Y^{*}, \quad C^{-1} M^{-1} \in B_{1}\{1\}
$$

Proof. Since $A$ is group invertible, we have that $C$ is nonsingular. Let $B=Y\left[\begin{array}{ll}B_{1} & B_{2} \\ B_{3} & B_{4}\end{array}\right] Y^{*}$, with $B_{1} \in \mathbb{C}^{r \times r}$.

If $A \overline{\leq}$, then $B=A A^{\oplus} B=B A^{\oplus} A=B A^{\oplus} B$ by Lemma 4.3. From

$$
A A^{\boxplus} B=Y\left[\begin{array}{cc}
I_{r} & 0 \\
0 & 0
\end{array}\right]\left[\begin{array}{ll}
B_{1} & B_{2} \\
B_{3} & B_{4}
\end{array}\right] Y^{*}=Y\left[\begin{array}{cc}
B_{1} & B_{2} \\
0 & 0
\end{array}\right] Y^{*},
$$

$B=A A^{\oplus} B$ and the above expression of $B$ we get that $B_{3}$ and $B_{4}$ are zero matrices. From

$$
B A^{\oplus} A=Y\left[\begin{array}{cc}
B_{1} & B_{2} \\
0 & 0
\end{array}\right]\left[\begin{array}{cc}
I_{r} & C^{-1} S \\
0 & 0
\end{array}\right] Y^{*}=Y\left[\begin{array}{cc}
B_{1} & B_{1} C^{-1} S \\
0 & 0
\end{array}\right] Y^{*}
$$

and $B=B A^{\oplus} A$ we get $B_{2}=B_{1} C^{-1} S$. From

$$
\begin{aligned}
B A^{\oplus B} B & =Y\left[\begin{array}{cc}
B_{1} & B_{2} \\
0 & 0
\end{array}\right]\left[\begin{array}{cc}
C^{-1} M^{-1} & 0 \\
0 & 0
\end{array}\right]\left[\begin{array}{cc}
B_{1} & B_{2} \\
0 & 0
\end{array}\right] \\
& =Y\left[\begin{array}{cc}
B_{1} C^{-1} M^{-1} B_{1} & B_{1} C^{-1} M^{-1} B_{2} \\
0 & 0
\end{array}\right] Y^{*}
\end{aligned}
$$

and $B A^{\oplus} B=B$ we get $B_{1}=B_{1} C^{-1} M^{-1} B_{1}$. Thus $B$ has the form in (4.12).

For the opposite implication, it is easy to check that $B=A A^{\oplus} B=B A^{\oplus} A=B A^{\oplus} B$, which gives $B \overline{\leq} A$ by Lemma 4.3 . 
The following lemma is obvious in view of Proposition 2.5.

Lemma 4.11. Let $A, B \in \mathbb{C}^{n \times n}$ be the same as in Theorem 4.10 with $B \overline{\leq} A$. Then $B_{1}$ is core invertible and $B^{\oplus}=Y\left[\begin{array}{cc}B_{1}^{\oplus} & 0 \\ 0 & 0\end{array}\right] Y^{*}$.

Proof. We write $B$ as in (4.12). The group invertibility of $B$ leads to the core invertibility of $B$ and $B_{1}$ by $\left[9\right.$, Theorem 1]. It is easy to verify that $B^{\oplus}=Y\left[\begin{array}{cc}B_{1}^{\oplus} & 0 \\ 0 & 0\end{array}\right] Y^{*}$ by using the Proposition 2.5.

Lemma 4.11 will useful in the next theorem. In the following, we will answer the following question: when the minus partial order is core partial order?

Theorem 4.12. Let $A, B \in \mathbb{C}^{n \times n}$ be core invertible. Assume that $A$ has the form (2.1). Then $B \stackrel{\oplus}{\leq} A$ if and only if $B$ can be written as

$$
B=Y\left[\begin{array}{cc}
B_{1} & B_{1} C^{-1} S \\
0 & 0
\end{array}\right] Y^{*}, \quad C^{-1} M^{-1} \in B_{1}\{1\}
$$

and $B_{1}=M C B_{1}^{\oplus} M C$.

Proof. Since $A$ is core invertible and the core invertibility is equivalent to the group invertibility, we get that $C$ is nonsingular. Let $B=Y\left[\begin{array}{ll}B_{1} & B_{2} \\ B_{3} & B_{4}\end{array}\right] Y^{*}$, where $B_{1} \in \mathbb{C}^{r \times r}$. Suppose $B \stackrel{\oplus}{\leq} A$. Since $B \stackrel{\oplus}{\leq} A$ implies $B \underset{\leq}{\leq} A$, Theorem 4.10 and Lemma 4.11, imply

$$
B=Y\left[\begin{array}{cc}
B_{1} & B_{1} C^{-1} S \\
0 & 0
\end{array}\right] Y^{*}, \quad B^{\oplus}=Y\left[\begin{array}{cc}
B_{1}^{\oplus} & 0 \\
0 & 0
\end{array}\right] Y^{*}, \quad C^{-1} M^{-1} \in B_{1}\{1\} .
$$

Since $B \stackrel{\oplus}{\leq} A$, by Lemma 4.4 , we know $A^{\oplus} B A^{\oplus}=B^{\oplus}$.

$$
\begin{aligned}
A^{\oplus} B A^{\oplus} & =Y\left[\begin{array}{cc}
C^{-1} M^{-1} & 0 \\
0 & 0
\end{array}\right]\left[\begin{array}{cc}
B_{1} & B_{2} \\
B_{3} & B_{4}
\end{array}\right]\left[\begin{array}{cc}
C^{-1} M^{-1} & 0 \\
0 & 0
\end{array}\right] Y^{*} \\
& =Y\left[\begin{array}{cc}
C^{-1} M^{-1} B_{1} & C^{-1} M^{-1} B_{2} \\
0 & 0
\end{array}\right]\left[\begin{array}{cc}
C^{-1} M^{-1} & 0 \\
0 & 0
\end{array}\right] Y^{*} \\
& =Y\left[\begin{array}{cc}
C^{-1} M^{-1} B_{1} C^{-1} M^{-1} & 0 \\
0 & 0
\end{array}\right] Y^{*} .
\end{aligned}
$$

From (??), (4.14) and $A^{\boxplus} B A^{\oplus}=B^{\oplus}$ ( by Lemma 4.4 ) we get

$$
C^{-1} M^{-1} B_{1} C^{-1} M^{-1}=B_{1}^{\oplus}
$$

That is $B_{1}=M C B_{1}^{\boxplus} M C$. The opposite inclusion is trivial. 


\section{Core invertibility under the core partial order}

In [13, Theorem 2.2], Mitra has shown that for matrices $A, B \in \mathbb{C}^{n \times n}$, if $A \stackrel{*}{\leq} B$, then $B^{\dagger}-A^{\dagger}=(B-A)^{\dagger}$. It is well-known that a complex matrix is Moore-Penrose invertible, but it is not true for the core inverse of a complex matrix. When we consider the CS decomposition in Lemma 2.2, then $A$ is core invertible if and only if $C$ is nonsingular and $A^{\oplus}=Y\left[\begin{array}{cc}C^{-1} M^{-1} & 0 \\ 0 & 0\end{array}\right] Y^{*}$. A natural question is that if we assume that $A$ and $B-A$ are both core invertible with $A \stackrel{\oplus}{\leq} B$, then $B$ is core invertible ? Moreover, if $B$ is core invertible, do we have $B^{\oplus}-A^{\oplus}=(B-A)^{\oplus}$ ? In the following theorem, we will answer this question.

Theorem 5.1. Let $A, B \in \mathbb{C}^{n \times n}$. Assume that $A$ has the form (2.1). If $A$ and $B-A$ are both core invertible and $A \stackrel{\oplus}{\leq} B$, then $B$ is core invertible. In this case

$$
B^{\oplus}=A^{\oplus}+(B-A)^{\oplus}-A^{\oplus} A(B-A)^{\oplus} .
$$

Proof. From Theorem 4.5, we have

$$
B-A=Y\left[\begin{array}{cc}
0 & 0 \\
0 & B_{4}
\end{array}\right] Y^{*}, \quad B_{4} \in \mathbb{C}^{(n-r) \times(n-r)} .
$$

Since $A$ and $B-A$ are both core invertible, we get $C$ is nonsingular and $B_{4}$ is core invertible in view of the Proposition 2.5. Thus $M C$ is core invertible and $(M C)^{\oplus}=C^{-1} M^{1}$. The equality (5.1) gives that

$$
B=Y\left[\begin{array}{cc}
M C & M S \\
0 & B_{4}
\end{array}\right] Y^{*}, \quad B_{4} \in \mathbb{C}^{(n-r) \times(n-r)}
$$

Let $X=Y\left[\begin{array}{cc}C^{-1} M^{1} & -C^{-1} S B_{4}^{\oplus} \\ 0 & B_{4}^{\oplus}\end{array}\right] Y^{*}$, we have

$$
\begin{aligned}
B X & =Y\left[\begin{array}{cc}
I & 0 \\
0 & B B_{4}^{\oplus}
\end{array}\right] Y^{*} \text { is Hermitian, } \\
X B^{2} & =Y\left[\begin{array}{ccc}
C^{-1} M^{-1} & -C^{-1} S B_{4}^{\oplus} \\
0 & B_{4}^{\oplus}
\end{array}\right]\left[\begin{array}{cc}
(M C)^{2} & M C M S+M S B_{4} \\
0 & \left(B_{4}\right)^{2}
\end{array}\right] Y^{*}=B, \\
B X^{2} & =Y\left[\begin{array}{cc}
I & 0 \\
0 & B B_{4}^{\oplus}
\end{array}\right]\left[\begin{array}{cc}
C^{-1} M^{-1} & -C^{-1} S B_{4}^{\oplus} \\
0 & B_{4}^{\oplus}
\end{array}\right] Y^{*}=X .
\end{aligned}
$$

Thus, $B^{\oplus}=X$ in view of Lemma 2.4.

That is we have $B^{\oplus}=Y\left[\begin{array}{cc}C^{-1} M^{-1} & -C^{-1} S B_{4}^{\oplus} \\ 0 & B_{4}^{\oplus}\end{array}\right] Y^{*}$. The equality (5.1) gives that $(B-A)^{\oplus}=Y\left[\begin{array}{cc}0 & 0 \\ 0 & B_{4}^{\oplus}\end{array}\right] Y^{*}$ in view of the Proposition 2.5. Thus $B^{\oplus}=A^{\oplus}+(B-A)^{\oplus}+$ $Y\left[\begin{array}{cc}0 & -C^{-1} S B_{4}^{\oplus} \\ 0 & 0\end{array}\right] Y^{*}$. Having in mind $A^{\oplus}=Y\left[\begin{array}{cc}C^{-1} M^{-1} & 0 \\ 0 & 0\end{array}\right] Y^{*}$. Finally, since we 
have $A^{\oplus} A=A A^{\#}$ and

$$
\begin{aligned}
Y\left[\begin{array}{cc}
0 & C^{-1} S B_{4}^{\oplus} \\
0 & 0
\end{array}\right] Y^{*} & =Y\left[\begin{array}{cc}
I_{r} & C^{-1} S \\
0 & 0
\end{array}\right]\left[\begin{array}{cc}
0 & 0 \\
0 & B_{4}^{\oplus}
\end{array}\right] Y^{*} \\
& =A A^{\#}(B-A)^{\oplus},
\end{aligned}
$$

Thus $B^{\oplus}=A^{\oplus}+(B-A)^{\oplus}-A^{\oplus} A(B-A)^{\oplus}$.

We will prove that $A^{2}=A B$ if and only if $Y\left[\begin{array}{cc}0 & C^{-1} S B_{4}^{\oplus} \\ 0 & 0\end{array}\right] Y^{*}=0$. Next we will show that $A A^{\#}(B-A)^{\oplus}=0$ if and only if $A^{2}=A B$. Since we have $B-A=$ $(B-A)^{\oplus}(B-A)^{2}$ and $(B-A)^{\oplus}=(B-A)\left((B-A)^{\oplus}\right)^{2}$, thus

$$
\begin{aligned}
A A^{\#}(B-A)^{\oplus}=0 & \Leftrightarrow A(B-A)^{\oplus}=0 \\
& \Leftrightarrow A(B-A)=0 .
\end{aligned}
$$

Corollary 5.2. Let $A, B \in \mathbb{C}^{n \times n}$. Assume that $A$ has the form (2.1). If $A$ and $B-A$ are both core invertible, $A \stackrel{\oplus}{\leq} B$, then $A^{2}=A B$ if and only if $B^{\oplus}-A^{\oplus}=(B-A)^{\boxplus}$.

In [2, Theorem 3.7], the author proved that if $A$ is an EP matrix, then $S=0$.

Corollary 5.3. Let $A, B \in \mathbb{C}^{n \times n}$. Assume that $A$ has the form (2.1). If $A$ and $B-A$ are both core invertible, $A \stackrel{\oplus}{\leq} B$ and $A$ is an EP matrix, then $B^{\oplus}-A^{\oplus}=(B-A)^{\oplus}$.

It is well-known that for complex matrices $A, B$ we have $A \stackrel{*}{\leq} B$ if and only if $A \overline{\leq} B$ and $(B-A)^{\dagger}=B^{\dagger}-A^{\dagger}$. For the core partial order, we also can get a similar result as follows.

Theorem 5.4. Let $A, B \in \mathbb{C}^{n \times n}$. Assume that $A$ has the form (2.1). If $A$ and $B-A$ are both core invertible, then $A \stackrel{\oplus}{\leq} B$ if and only if $A \leq B$ and $B$ is core invertible with

$$
B^{\oplus}-A^{\oplus}=\left(I_{n}-A^{\oplus} A\right)(B-A)^{\oplus} .
$$

Proof. By (??), we can get that $A^{\oplus} A=Y\left[\begin{array}{cc}I_{r} & C^{-1} S \\ 0 & 0\end{array}\right] Y^{*}$. From the proof of Theorem 5.1 , it is enough to prove that $A \leq B$ and $B$ is core invertible with $B^{\oplus}-A^{\oplus}=\left(I_{n}-\right.$ $\left.A^{\oplus} A\right)(B-A)^{\oplus}=Y\left[\begin{array}{cc}0 & -C^{-1} S B_{4}^{\oplus} \\ 0 & B_{4}^{\oplus}\end{array}\right] Y^{*}$ implies that $A \stackrel{\oplus}{\leq} B$. Since

$$
\begin{aligned}
B^{\oplus} A B^{\oplus} & =\left[A^{\oplus}+Y\left[\begin{array}{cc}
0 & -C^{-1} S B_{4}^{\oplus} \\
0 & B_{4}^{\oplus}
\end{array}\right] Y^{*}\right] A\left[A^{\oplus}+Y\left[\begin{array}{cc}
0 & -C^{-1} S B_{4}^{\oplus} \\
0 & B_{4}^{\oplus}
\end{array}\right] Y^{*}\right] \\
& =Y\left[\begin{array}{cc}
I_{r} & -C^{-1} S \\
0 & 0
\end{array}\right] Y^{*}\left[A^{\oplus}+Y\left[\begin{array}{cc}
0 & -C^{-1} S B_{4}^{\oplus} \\
0 & B_{4}^{\oplus}
\end{array}\right] Y^{*}\right] \\
& =Y\left[\begin{array}{cc}
C^{-1} M^{-1} & 0 \\
0 & 0
\end{array}\right] Y^{*}=A^{\oplus} .
\end{aligned}
$$

Thus $B^{\oplus} A B^{\oplus}=A^{\oplus}$, which gives that $A \stackrel{\oplus}{\leq} B$ in view of [16, Theorem 4.10]. 
Theorem 5.5. Let $A, B \in \mathbb{C}^{n \times n}$. Assume that $A$ has let $A$ have the form (2.1) and $A$ is core invertible. If $B=Y\left[\begin{array}{cc}M C & M S \\ 0 & B_{4}\end{array}\right] Y^{*}$ with $B_{4}$ is core invertible and $S B_{4}=0$, then

(1) If $A \stackrel{\oplus}{\leq} B$, then $B-A$ is core invertible and $(B-A)^{\oplus}=B^{\oplus}-A^{\boxplus}$;

(2) $A \stackrel{\oplus}{\leq} B$ if and only if $(B-A) \stackrel{\oplus}{\leq} B$.

Proof. The part (1) is a corollary of Theorem 5.1.

The part (2), suppose $A \stackrel{\circledast}{\leq} B$. It is sufficient to show that $(B-A)^{*}(B-A)=(B-A)^{*} B$ and $(B-A)^{2}=B(B-A)$ by [16, Theorem 2.4].

$$
\begin{aligned}
& (B-A)^{*}(B-A)=Y\left[\begin{array}{cc}
0 & 0 \\
0 & B_{4}^{*}
\end{array}\right]\left[\begin{array}{cc}
0 & 0 \\
0 & B_{4}
\end{array}\right] Y^{*}=Y\left[\begin{array}{cc}
0 & 0 \\
0 & B_{4}^{*} B_{4}
\end{array}\right] Y^{*} ; \\
& (B-A)^{*} B=Y\left[\begin{array}{cc}
0 & 0 \\
0 & B_{4}^{*}
\end{array}\right]\left[\begin{array}{cc}
M C & M S \\
0 & B_{4}
\end{array}\right] Y^{*}=Y\left[\begin{array}{cc}
0 & 0 \\
0 & B_{4}^{*} B_{4}
\end{array}\right] Y^{*} \\
& (B-A)^{2}=Y\left[\begin{array}{cc}
0 & 0 \\
0 & B_{4}
\end{array}\right]\left[\begin{array}{cc}
0 & 0 \\
0 & B_{4}
\end{array}\right] Y^{*}=Y\left[\begin{array}{cc}
0 & 0 \\
0 & B_{4}^{2}
\end{array}\right] Y^{*} \\
& B(B-A)=Y\left[\begin{array}{cc}
M C & M S \\
0 & B_{4}
\end{array}\right]\left[\begin{array}{cc}
0 & 0 \\
0 & B_{4}
\end{array}\right] Y^{*}=Y\left[\begin{array}{cc}
0 & 0 \\
0 & B_{4}^{2}
\end{array}\right] Y^{*}
\end{aligned}
$$

From $(5.3),(5.4),(5.5)$ and $(5.6)$ we get $(B-A) \stackrel{\oplus}{\leq} B$. Conversely, it is easy to check that $A^{*} A=A^{*} B$ and $A^{2}=B A$.

\section{ACKNOWLEDGMENTS}

This research is supported by the National Natural Science Foundation of China (No. 11201063 and No. 11371089), the Natural Science Foundation of Jiangsu Province (No. BK20141327). The first author is grateful to China Scholarship Council for giving him a purse for his further study in Universidad Politécnica de Valencia, Spain.

\section{References}

[1] O.M. Baksalary, G. Trenkler. Core inverse of matrices. Linear Multilinear Algebra 58(6) (2010), 681-697.

[2] J. Benítez, A new decomposition for square matrices, Electron. J. Linear Algebra 20(2010), 207-225.

[3] J. Benítez, X.J. Liu, A short proof of a matrix decomposition with applications, Linear Algebra Appl. 438 (2013), 1398-1414.

[4] R.E. Cline and R.E. Funderlic. The rank of a difference of matrices and associated generalized inverses. Linear Algebra and its Applications, 24 (1979), 185-215.

[5] C.G. Cao, Some results of group inverses for partitioned matrices over skew fields, Journal of Natural Science of Heilongjiang University, 18(3) (2001), 5-7. 
[6] M.P. Drazin, Natural structures on semigroups with involution, Bull. Amer. Math. Soc. 84(1) (1978), 139-141.

[7] R.E. Hartwig, How to order regular elements ?, Math. Japon. 25 (1980), 1-13.

[8] R.E. Hartwig, K. Spindelböck. Matrices for which $A^{*}$ and $A^{\dagger}$ commute. Linear Multilinear Algebra 14 (1984), 241-256.

[9] R.E. Hartwig, J. Shoaf, Group inverses and Drazin inverses of bidiagonal and triangular toeplitz matrices, J. Austral. Math. Soc. 24 (1977), 10-34.

[10] L. Lebtahi, P. Patrićio, N. Thome, The diamond partial order in rings, Linear Multilinear Algebra 62(3) (2013), 386-395.

[11] K. Manjunatha Prasad, K.S. Mohana, Core-EP inverse, Linear Multilinear Algebra 62(6) (2014), 792-802.

[12] G. Marsaglia and G.P.H. Styan. Equalities and inequalities for ranks of matrices. Linear and Multilinear Algebra, 2 (1974), 269-292.

[13] S.K. Mitra, On group inverses and the sharp order, Linear Algebra Appl. 92 (1987), 17-37.

[14] S.K. Mitra, P. Bhimasankaram, S.B. Malik, Matrix Partial Orders, Shorted Operators and Applications, World Scientific, 2010.

[15] D.S. Rakić, Nebojša Č. Dinčić, D.S. Djordjević. Group, Moore-Penrose, core and dual core inverse in rings with involution. Linear Algebra Appl. 463 (2014), 115-133.

[16] D.S. Rakić, D.S. Djordjević, Star, sharp, core and dual core partial order in rings with involution, Appl. Math. Comput. 259 (2015), 800-818.

[17] J. von Neumann. On regular rings. Proc. Nati. Acad. Sci. U.S.A. 22(12) (1936), 707713.

[18] H.X. Wang, Core-EP decomposition and its applications, Linear Algebra Appl. 508 (2016), 289-300.

[19] H.K. Wimmer, Canonical angles of unitary spaces and perturbations of direct complements, Linear Algebra Appl. 287 (1999) 373-379.

[20] S.Z. Xu, J.L. Chen, J. Benítez. EP elements in rings with involution. arXiv:1602.08184[math.RA].

[21] S.Z. Xu, J.L. Chen, X.X. Zhang, New characterizations for core inverses in rings with involution, Front. Math. China. 12(1) (2017), 231-246.

[22] X.X. Zhang, S.Z. Xu, J.L. Chen, Core partial order in rings with involution, arXiv:1601.03120[math.RA]. 